\title{
MECHANICAL BEHAVIOUR AND PHASE TRANSITION MECHANISMS OF A SHAPE MEMORY ALLOY BY MEANS OF A NOVEL ANALYTICAL MODEL
}

\author{
Andrea CARPINTERI", Vittorio DI COCCO", Giovanni FORTESE*, Francesco IACOVIELLO**, Stefano NATALI"*; \\ Camilla RONCHEI*, Daniela SCORZA*, Sabrina VANTADORI*, Andrea ZANICHELLI*
}

\author{
Department of Engineering and Architecture, University of Parma, Parco Area delle Scienze 181/A, 43124 Parma, Italy \\ ** Department of Civil and Mechanical Engineering University of Cassino and Lazio Meridionale, Via G. Di Biasio 43, 03043 Cassino (FR), Italy \\ ${ }^{* * *}$ Department Chemical Engineering Materials Environment, University of Roma "La Sapienza”, via Eudossiana 18, 00184 Rome, Italy \\ andrea.carpinteri@unipr.it, v.dicocco@unicas.it, giovanni.fortese@studenti.unipr.it, iacoviello@unicas, stefano.natali@uniroma1.it, \\ camilla.ronchei@nemo.unipr.it, daniela.scorza@unipr.it, sabrina.vandatori@unipr.it, andrea.zanichelli1@studenti.unipr.it
}

received 19 April 2017, revised 6 June 2018, accepted 8 June 2018

\begin{abstract}
The aim of the present paper is to examine both the fatigue behaviour and the phase transition mechanisms of an equiatomic pseudo-elastic NiTi Shape Memory Alloy through cyclic tests (up to 100 loading cycles). More precisely, miniaturised dog-bone specimens are tested by using a customised testing machine and the contents of both austenite and martensite phase are experimentally measured by means of X-Ray diffraction (XRD) analyses. On the basis of such experimental results in terms of martensite content, an analytical model is here formulated to correlate the stress-strain relationship to the phase transition mechanisms. Finally, a validation of the present model by means of experimental data pertaining the stress-strain relationship is performed.
\end{abstract}

Key words: Fatigue Behaviour, Phase Transition Mechanisms, Shape Memory Alloy, Tensile Cyclic Test, X-Ray Diffraction Analyses

\section{INTRODUCTION}

Shape memory alloys (SMAs) are an important class of materials since they are able to recover the initial shape thanks to reversible phase transition mechanisms, even after severe deformations.

The near equiatomic Nickel-Titanium binary system (NiTi) belongs to the class of SMAs (Shimamoto et al., 2004; Otuska and Ren, 2005; Li et al., 2009). Large deformations induced in such alloys are perfectly recovered by two possible paths:

- such alloys spontaneously return to the original predeformation appearance when heated at a characteristic temperature (shape memory effect);

- such alloys recover the original pre-deformation appearance by simply removing the mechanical load (pseudoelasticity or superelasticity).

The explanation of these peculiar behaviours can be found in the crystallography and thermodynamics of SMAs (Li et al., 2009).

Due to the above unique features, NiTi alloys are currently used in mechanical, civil and medical field(Kuribayashi et al., 2006), and the use of NiTi alloys is expected to rise due to a continuous improvement (Bujoreanu, 2008).

In last decades, several experimental tests have been performed in order to better understand the thermo-mechanical properties of such alloys, and some analytical and numerical models have been developed to describe their mechanical and functional behaviour (that is, the microstructural transition from austenite to martensite and vice-versa). In particular, the scientific community has agreed that crack initiation and propagation are significantly affected by the phase transition mechanisms and, consequently, NiTi alloys exhibit unusual fatigue and fracture responses with respect to common alloys (Shimamoto et al., 2004). Experimental studies have recently been performed for both static (Maletta et al., 2013) and cyclic loading conditions (Robertson et al., 2007; Gall et al., 2008). Furthermore, numerical studies have been carried out by using both standard finite element codes (Maletta et al., 2009) and special constitutive models for SMAs (Freed and Banks-Sills, 2001). Finally, many analytical models have been proposed (Maletta and Furgiuele, 2010; Baxevanis and Lagoudas, 2012; Malett, 2012).

The aim of the present paper is to examine both the fatigue behaviour and the phase transition mechanisms of an equiatomic pseudo-elastic NiTi Shape Memory Alloy through cyclic tests (up to 100 loading cycles). Miniaturised dog-bone specimens are tested by using a customised testing machine, and the contents of both austenite and martensite phase are experimentally measured by means of X-Ray diffraction (XRD) analyses.

On the basis of such experimental results, an analytical model is here formulated to correlate the stress-strain relationship to the phase transition mechanisms. The model assumes that the fatigue behaviour of the system (that is, phases and test machine) can be schematised as that of one spring, or two springs arranged in series or in parallel. Finally, a validation of the present model by means of experimental data pertaining the stress-strain relationship is performed.

\section{EXPERIMENTAL CAMPAIGN}

An equiatomic pseudo-elastic NiTi alloy, which exhibits a pseudo elastic behaviour, is made in the laboratory by using a vacuum furnace. The miniaturised specimens are characterised by a flat dog-bone shape and the geometrical sizes are reported in Fig. 1.

The tensile cyclic tests are performed by means of a custom- 


\section{sciendo}

Andrea Carpinteri, Vittorio Di Cocco, Giovanni Fortese, Francesco lacoviello, Stefano Natali, Camilla Ronchei, Daniela Scorza, Sabrina Vantadori, Andrea Zanichelli Mechanical Behaviour and Phase Transition Mechanisms of a Shape Memory Alloy by Means of a Novel Analytical Model

ised testing machine, which allows in situ XRD measurements (details can be found in Di Cocco et al. (2014a, b).

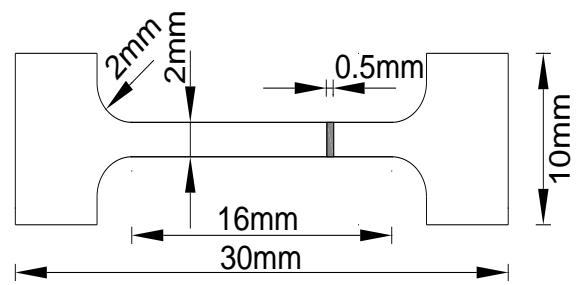

Fig. 1. Geometry miniaturised flat dog-bone specimen.

In particular, a diffractometer equipped with a vertical powder goniometer is employed in order to perform the XRD analyses. Moreover, the PowderCell software allows to determine both the theoretical diffractograms and the structure models (details on the XRD analyses are provided in Di Cocco et al. (2014a).

In order to carry out the XRD analyses at fixed values of the applied deformation, the testing machine is equipped with a removable loading frame and two load cells Leane FGP 1000. Since no strain gauges are used, a linear variable differential transducer is employed in order to measure the whole elongation of the specimen.

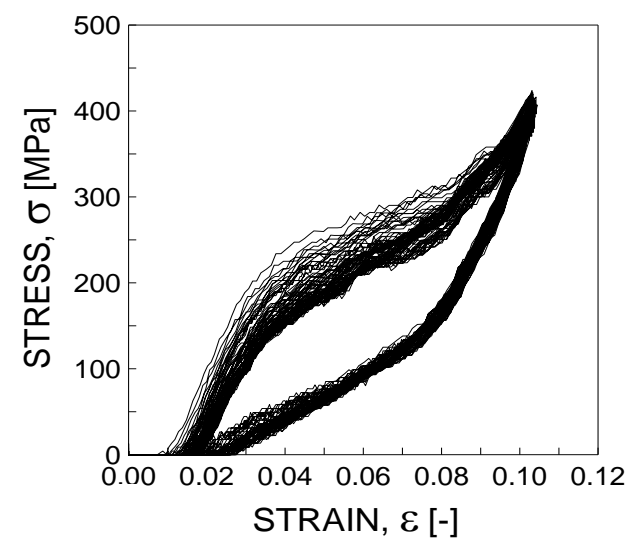

Fig. 2. Experimental fatigue behaviour: stress- strain relationship

Each tensile cyclic test is performed step by step under displacement control of the crosshead until a number of loading cycles equal to 100 cycles is reached.

For each cycle, the phases content is experimentally determined at ten fixed values of strain (named strain steps in the following) by means of XRD analyses.

The experimental relationship between stress, $\sigma$, and strain, $\varepsilon$, is reported in Fig. 2.

For each of the aforementioned steps, the XRD analyses allow to quantify the phase transition mechanisms through the diffraction spectra. For instance, considering the initial loading condition $(\varepsilon=0)$, the diffraction spectrum corresponds to the austenite phase (cubic lattice cell), whereas the diffraction spectrum related to $\varepsilon=0.1$ corresponds to the martensite phase (monocline lattice cell). Moreover, from the experimental results, it is in general observed that both the austenite-martensite transition in the loading condition and the martensite-austenite transition in the unloading condition are not characterised by a linear trend.

The intensity of austenitic and martensitic peaks allows to quantify the contents of both austenite and martensite. For a given value of strain $\varepsilon$, the content of austenite is proportional to the maximum amplitude of austenite peak in undeformed initial condition, corresponding to fully austenitic structure.

\section{ANALYTICAL MODEL}

The fatigue behaviour of the NiTi SMA tested under tensile cyclic loading is hereafter described by means of a novel analytical model. In particular, the stress-strain relationship is defined in an analytical way as a function of the phase transition mechanisms (from austenite to martensite and vice-versa).

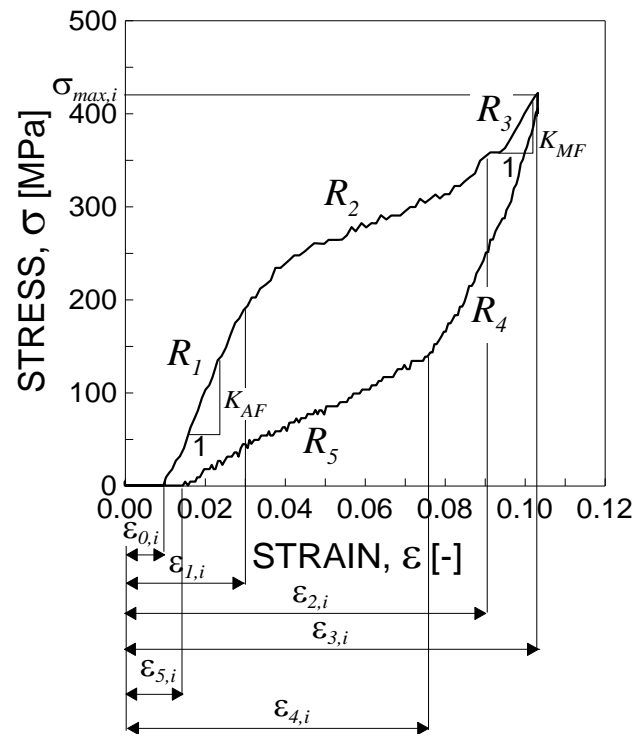

Fig. 3. Five regions characterised by different contents of austenite and martensite in the stress-strain diagram of the i-th loading cycle

On the basis of the experimental results presented in Section 2 , it is possible to define five regions, characterised by different contents of austenite and martensite, in the stress-strain diagram (Fig. 2). For the $i$-th cycle (with $i=1, \ldots, 100$ ), the following five regions can be identified (see Fig. 3):

1. Region $1\left(\varepsilon_{0, \mathrm{i}} \leq \varepsilon \leq \varepsilon_{1, i}, R_{1}\right.$ in Fig. 3): the content of the austenite phase is predominant and approximately constant with increasing $\varepsilon$;

2. Region $2\left(\varepsilon_{1, i} \leq \varepsilon \leq \varepsilon_{2, i}, R_{2}\right.$ in Fig. 3): the content of austenite phase decreases while the content of martensite phase increases with increasing $\varepsilon$;

3. Region $3\left(\varepsilon_{2, i} \leq \varepsilon \leq \varepsilon_{3, i} R_{3}\right.$ in Fig. 3): the content of the martensite phase is predominant and approximately constant with increasing $\varepsilon$;

4. Region $4\left(\varepsilon_{4, i} \leq \varepsilon \leq \varepsilon_{3, i} R_{4}\right.$ in Fig. 3): the content of the martensite phase is predominant and approximately constant with decreasing $\varepsilon$;

5. Region $5\left(\varepsilon_{5, i} \leq \varepsilon \leq \varepsilon_{4, i} R_{5}\right.$ in Fig. 3): the content of martensite phase decreases while the content of austenite phase increases with decreasing $\varepsilon$.

Since the values of austenite and martensite are experimentally measured only at 1,50 and 100 loading cycles, a diffusion model is here employed to estimate the phase content as a function of the strain $\varepsilon$, for each $i$-th cycle. The content of martensite, $M$, is given by the following expression: 


$$
M(\%)=\frac{100}{1+a \cdot \exp (-b \cdot \varepsilon)}
$$

where $a$ is equal to 700 and 70 for loading and unloading condition, respectively. Moreover, $b$ is a parameter depending on the number $i$ of cycles (with $i \geq 1$ ), defined as follows:

$$
b=-4.2 \ln (i)+1
$$

The content of austenite phase, $A$, is computed by using the following equation:

$$
A(\%)=100-M(\%)
$$

The aforementioned five regions are characterised by different limit values of $\varepsilon$, which are defined for the $i$-th cycle as follows:

1. $\varepsilon_{0, i}$ is computed according to the following expression, obtained from a best-fit procedure by considering the experimental values of $\varepsilon_{0, i}$ for $i=1.50$ and 100 loading cycles:

$$
\varepsilon_{0, i}=0.009763 \cdot(i)^{0.1525}
$$

2. $\varepsilon_{1, i}$ is determined recalling Eqs (1) and (2) and assuming $a=700, \quad i=1$ and $M=3 \%$, being $\varepsilon_{1, i}$ almost independent of the number $i$ of cycles (Figs. 2 and 3 ): $\varepsilon_{1, i}=\varepsilon_{1}=0.030750$;

3. $\varepsilon_{2, i}$ is determined recalling Eqs (1) and (2) and assuming $a=700, \quad i=1$ and $M=95 \%$, being $\varepsilon_{2, i}$ almost independent of the number $i$ of cycles (Figs 2 and 3 ): $\varepsilon_{2, i}=\varepsilon_{2}=0.094955$

4. $\varepsilon_{3, i}$ is determined recalling Eqs (1) and (2) and assuming $a=700, \quad i=1$ and $M=98 \%$, being $\varepsilon_{3, i}$ almost independent of the number $i$ of cycles (see Figs 2 and 3): $\varepsilon_{3, i}=\varepsilon_{3}=0.104429$;

5. $\varepsilon_{4, i}$ is determined recalling Eqs (1) and (2) and assuming $a=70, \quad i=1$ and $M=98 \%$, being $\varepsilon_{4, i}$ almost independent of the number $i$ of cycles (Figs 2 and 3): $\varepsilon_{4, i}=\varepsilon_{4}=0.081280$;

6. $\varepsilon_{5, i}$ is computed according to the following expression, obtained from a best-fit procedure by considering the experimental values of $\varepsilon_{5, i}$ for $i=1.50$ and 100 loading cycles:

$$
\varepsilon_{5, \mathrm{i}}=0.000158 \cdot(i)+0.010990
$$

Considering the above limit values of $\varepsilon$, an analytical model is hereafter formulated in order to describe the relationship between stress and strain of the NiTi SMA. In particular, the fatigue behaviour is analytically interpreted as a system of springs characterised by a stiffnesses depending on the phase transition mechanism, and more precisely:

a) Region 1 (loading condition). The stress-strain relationship is characterised by a linear trend and the fatigue behaviour of the system is schematised as that of one spring with a stiffness equal to $K_{A F}$ :

$$
\sigma_{1, i}(\varepsilon)=K_{A F} \cdot\left(\varepsilon-\varepsilon_{0, i}\right)
$$

where $\varepsilon_{0, i}$ is computed through Eq. (4). The stiffness $K_{A F}$ is experimentally measured (Fig. 3), and corresponds to that offered by the austenite phase, being the content of martensite approximately equal to zero in this region;

b) Region 3 (loading condition). The stress-strain relationship is characterised by a linear trend and the fatigue behaviour of the system is schematised as that of one spring with a stiff- ness equal to $K_{M F}$ :

$$
\sigma_{3, i}(\varepsilon)=K_{M F} \cdot\left(\varepsilon-\varepsilon_{3}\right)+\sigma_{\max , i}
$$

where the dependence of $\sigma_{\max , i}$ from $i$ is obtained by means of a best-fit procedure, by considering the experimental values of $\sigma_{\max , i}$ for $i=1.50$ and 100 loading cycles:

$\sigma_{\max , i}=421.92 \cdot(i)^{-0.0088}$

The stiffness $K_{M F}$ is experimentally measured (Fig. 3), and corresponds to that offered by the martensite phase, being the content of austenite approximately equal to zero in this region;

c) Region 2 (loading condition). The stress-strain relationship is characterised by a non-linear trend and the fatigue behaviour of the system is schematised as that of two springs in series with stiffness depending on both $\varepsilon$ and the number $i$ of loading cycles:

$\sigma_{2, i}(\varepsilon)=\frac{1}{\frac{1}{K_{A F, i}(\varepsilon)}+\frac{1}{K_{M F, i}(\varepsilon)}} \cdot \varepsilon+D_{i}=$

$\left[A_{i} \varepsilon^{2}+B_{i} \varepsilon+C_{i}\right] \cdot \varepsilon+D_{i}$

where the dependence of $A_{i}$ and $B_{i}$ on $i$ are obtained through a best-fit procedure by considering the experimental values of $A_{i}$ and $B_{i}$, for $i=1.50$ and 100 loading cycles:

$A_{i}=390.625 \cdot(i)^{2}-36719.659 \cdot(i)+$ 1829350.489

$B_{i}=-72.773 \cdot(i)^{2}+6975.84 \cdot(i)-$

343752.288

Moreover, the crossing conditions of $\sigma_{2, i}$ by the two points $\left(\varepsilon_{1}, \sigma_{1, i}\left(\varepsilon_{1}\right)\right)$ and $\left(\varepsilon_{2}, \sigma_{3, i}\left(\varepsilon_{2}\right)\right)$ are employed in order to determine the coefficients $C_{i}$ and $D_{i}$;

d) Region 4 (unloading condition). The stress-strain relationship is characterised by a linear trend and the fatigue behaviour of the system is schematised as that of two springs in parallel with constant stiffness:

$\sigma_{4, i}(\varepsilon)=\left(K_{M F}+K_{m}\right) \cdot \varepsilon+F_{i}$

where $K_{m}$ is the testing machine stiffness, experimentally measured and equal to $6227.57 \mathrm{MPa}$. Moreover, the crossing condition of $\sigma_{4, i}$ by the point $\left(\varepsilon_{3}, \sigma_{\text {max }, i}\right)$ is employed in order to determine the coefficient $F_{i}$;

e) Region 5 (unloading condition). The stress-strain relationship is characterised by a linear trend and the fatigue behaviour of the system is schematised as that of two springs in series with stiffness depending on both $\varepsilon$ and the number $i$ of loading cycles:

$\sigma_{5, i}(\varepsilon)=\frac{1}{\frac{1}{K_{A F, i}(\varepsilon)}+\frac{1}{K_{M F, i}(\varepsilon)}} \cdot \varepsilon+H_{i}=G_{i} \cdot \varepsilon+H_{i}$

where the coefficients $G_{i}$ and $H_{i}$ are determined through crossing conditions of $\sigma_{5, i}$ by the two points $\left(\varepsilon_{4}, \sigma_{4, i}\left(\varepsilon_{4}\right)\right)$ and $\left(\varepsilon_{5, i}, 0\right)$.

Finally, the relationship between stress and strain for each value of $i$ (that is, for $i=1, \ldots, 100$ cycles) is computed through Eqs (1) - (12), where the strain limit values of the five above regions are computed according to Eqs (1),(2) for each cycle. 
Andrea Carpinteri, Vittorio Di Cocco, Giovanni Fortese, Francesco lacoviello, Stefano Natali, Camilla Ronchei, Daniela Scorza, Sabrina Vantadori, Andrea Zanichelli

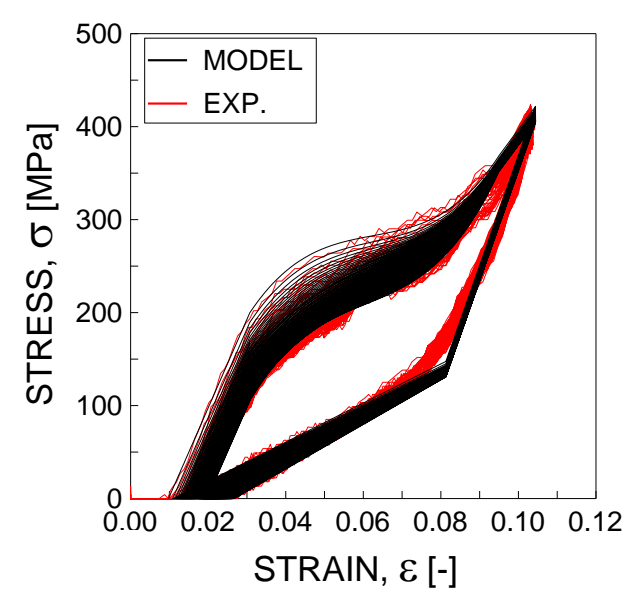

Fig. 4. Analytical and experimental results in terms of stress- strain relationship

In Fig. 4, the analytical stress-strain relationship is plotted together with the experimental stress-strain results. The analysis of the results indicates that the agreement between experimental and theoretical stress-strain relationship is satisfactory,

On the basis of such encouraging results, we can remark that the present model seems to be a promising engineering tool, able to describe the relationship between the fatigue behaviour and the transition phase mechanisms of SMAs with an adequate accuracy.

\section{CONCLUSIONS}

In the present paper, the fatigue behaviour and the phase transition mechanisms of a NiTi SMA have been examined by means of cyclic tests (up to 100 loading cycles). Miniaturised dog-bone specimens have been tested by using a customised testing machine, and the contents of both austenite and martensite phase have been experimentally measured by means of $X$ Ray diffraction (XRD) analyses.

On the basis of such experimental results in terms of martensite content, an analytical model has been formulated to correlate the stress-strain relationship to the phase transition mechanisms (in terms of martensite content). Five regions, characterised by different contents of austenite and martensite phase, have been identified in the stress-strain curve, for each cycle. For each of such regions, the stress - strain relationship has analytically been formulated, and the fatigue behaviour of the NiTi SMA has been evaluated.

Although the agreement between analytical and experimental results in term of stress-strain relationship has been satisfactory, further experimental tests need to be performed in order to develop a robust procedure appropriate for practical applications.

\section{REFERENCES}

1. Baxevanis T., Lagoudas D. (2012), A mode I fracture analysis of a center-cracked in niti shape memory alloy panel under plane stress, International Journal of Fracture, 175, 151-166.

2. Bujoreanu L.G. (2008), On the influence of austenitization on the morphology of alfa-phase in tempered $\mathrm{Cu}-\mathrm{Zn}-\mathrm{Al}$ shape memory alloys, Materials Science and Engineering A, 481, 395-403.

3. Di Cocco V., lacoviello F., Maletta C., Natali S. (2014a), Cyclic microstructural transitions and fracture micromechanisms in a near equiatomic NiTi alloy, International Journal of Fatigue, 58, 136-143.

4. Di Cocco V., lacoviello F., Natali S., Volpe V. (2014b), Fatigue crack behavior on a Cu-Zn-Al SMA, Frattura ed Integrità Strutturale, 30, 454-461.

5. Freed Y., Banks-Sills L. (2001), Crack growth resistance of shape memory alloys by means of a cohesive zone model, Journal of the Mechanics and Physics of Solids, 55, 2157-2180.

6. Gall K., Tyber J., Wilkesanders G., Robertson S.W., Ritchie R.O., Maier H.J. (2008), Effect of microstructure on the fatigue of hotrolled and cold-drawn NiTi shape memory alloys, Materials Science and Engineering A, 486, 389-403.

7. Kuribayashi K., Tsuchiya K., You Z., Tomus D., Umemoto M., Ito T., Sasaki M. (2006), Self-deployable origami stent grafts as a biomedical application of Ni-rich TiNi shape memory alloy foil, Materials Science and Engineering A, 419, 131-137.

8. Li Y.F., Mi X.J., Tan J., Gao B.D. (2009), Thermo-mechanical cyclic transformation behavior of Ti-Ni shape memory alloy wire, Materials Science and Engineering A, 509, 8-13.

9. Maletta C. (2012), A novel fracture mechanics approach for shape memory alloys with trilinear stress-strain behavior, International Journal of Fracture, 177, 39-51.

10. Maletta C., Falvo A., Furgiuele F., Leonardi A. (2009), Stress induced martensitic transformation in the crack tip region of a $\mathrm{NiTi}$ alloy, Journal of Materials Engineering and Performance, 18, 679-685.

11. Maletta C., Furgiuele F. (2010), Analytical modeling of stress induced martensitic transformation in the crack tip region of nickeltitanium alloys, Acta Materialia, 58, 92-101.

12. Maletta C., Furgiuele F., Sgambitterra E. (2013), Crack tip stress distribution and stress intensity factor in shape memory alloys, Fatigue and Fracture of Engineering Materials and Structures, 36, 903-912.

13. Otsuka K., Ren X. (2005), Physical metallurgy of Ti-Ni-based shape memory alloys, Progress in Materials Science, 50, 511-678.

14. Robertson S.W., Mehta A., Pelton A.R., Ritchie R.O. (2007), Evolution of crack-tip transformation zones in superelastic Nitinol subjected to in situ fatigue: a fracture mechanics and synchrotron Xray microdiffraction analysis, Acta Materialia, 55, 6198-6207.

15. Shimamoto A., Zhao H.Y., Abe H. (2004), Fatigue crack propagation and local crack-tip strain behavior in TiNi shape memory fiber reinforced composite, International Journal of Fatigue, 26, 533-542. 